$\xi=1$ 国

\title{
Assessment and Analysis of Software Reliability Using Machine Learning Techniques
}

\author{
G.Krishna Mohan ${ }^{1}$, N.Yoshitha ${ }^{2}$, M.L.N.Lavanya ${ }^{3}$, A.Krishna Priya ${ }^{4}$ \\ ${ }^{1,2,3,4}$ Department of CSE, K L E F, Guntur, India-522502. \\ *Corresponding author E-mail: gvlkm@kluniversity.in
}

\begin{abstract}
Software reliability models access the reliability by fault prediction. Reliability is a real world phenomenon with many associated real time problems and to obtain solutions to problems quickly, accurately and acceptably a large no. of soft computing techniques has been developed. We attempt to address the software failure problems by modeling software failure data using the machine learning techniques such as support vector machine (SVM) regression and generalized additive models. The study of software reliability can be categorized into three parts: modeling, measurement, improvement. Programming unwavering quality demonstrating has developed to a point that important outcomes can be acquired by applying appropriate models to the issue; there is no single model all inclusive to every one of the circumstances. We propose different machine learning methods for the evaluation of programming unwavering quality, for example, artificial neural networks, support vector machine calculation approached. We at that point break down the outcomes from machine getting the hang of demonstrating, and contrast them with that of some summed up direct displaying procedures that are proportional to programming dependability models.
\end{abstract}

Keywords:Software-reliability, artificial neural networks, Support vector machine, Machine learning techniques.

\section{Introduction}

The main purpose of this report is to propose process of hiring quality software which involves in determination of software reliability qualitative indices mainly based on machine learning techniques. Since the universe is taking on the techniques that are not explicitly being programmed and how long, the technique is trustworthy in the usage of the software is given away here. Here the problem is even by knowing different machine learning techniques, by evaluating software quality and by knowing which technique the best fit for what purpose and implementation of those is plays important roles. Achieve hundred percent quality is not a childlike thing; hence It is similarly hard to make the infrastructure necessary to measure software quality. Each one of the technique has its own importance and better means of execution, but the good implementation leads good results. Most of the people believe that they cannot afford to have resources spend any time on creating the processes, but these are either way simple or bold in understanding and in the implementation and have optimistic ways to report on software quality measurement. In this we are mainly focusing on two famous techniques called Support Vector machines (SVM) and Artificial Neural Network (ANN) how are they related and how far they evolved.

Software Reliability is a technique which reduces the possibility of an error-free software operation of a designated period in a modified environment. Software Reliability is also a major factor bearing on system dependability. Applying the term reliability for the extreme Machine learning techniques. Machine learning is an automated data processing system that is based along with some set of simple, fixed rules and the system can learn from incoming data. The difference between machine learning and explicit pro- gramming is accurately defining machine learning is a single sentence between very, very hard, but the point is- The machine can learn (how to reach decisions) from data alone without asking a human expert to tell the machine exactly what to use. Of course, a self- learning machine has to be programmed as well. Machine learning algorithms do not care about the problem you are solving. they are generic such that they extend across many problem areas where the traditional explicit programming is typically very domain specific. The uses of machine learning techniques have indicated amazing upgrades for the pre-expression of programming dependability than customary measurable methods. In this report, we utilize some notable machine learning strategies, for example, manufactured neural net-works, bolster vector machines, course relationship neural system, choice trees and fluffy derivation framework to foresee the dependability of a product item. The proposed models are to be measured utilizing the parameters like mean absolute error, root mean squared blunder, connection coefficient.

\section{Literature Survey}

Interestingly by picking the Several ML systems have been anticipated and used in the writing for programming unwavering quality demonstrating and expectation. A portion of the methods are hereditary programming, quality articulation programming, manufactured neural system, choice trees, support vector machines, sustain forward neural net, fluffy models, summed up neural system, and so on. (Karunanithi, 2015) did investigation of nitty gritty branch of knowledge to clarify the utilization of connectionist models in the unwavering quality development expectation for the product. Concentrated on the improvement of 
fluffy programming dependability models rather than probabilistic programming unwavering quality models as he says that dependability is fluffy in nature. Adjusted from Ho et al. [17] completed a thorough investigation of connectionist models and their relevance to programming dependability expectation and gathered that these are more extreme when contrasted with customary models. (Sun and Huang, 2006) had connected neural system for anticipating programming unwavering quality. (Madsen, 2016) concentrated on the use of delicate registering procedures for programming unwavering quality expectation. (Cove and Hong, 2006) performed tests utilizing SVMs for determining programming unwavering quality. Regardless of late advances in this field, it was remarked that distinctive models have changed prescient unwavering quality capacities. As indicated by (Wang, 2005) "SVM's have been taken in the save arrange for the improvement of neural systems (NN's). SVM advanced from the sound hypothesis to the doing and analyze, while the NN's taken after more heuristic frame application and broad experimentation to the hypothesis. "As indicated by (Sunken,2002) "While the weight rot term is a critical aspect in getting great speculation with regards to neural system for relapse, the edge assume a comparative part in grouping issue." In contrasting and customary multilayer perception neural systems that bear from the presence of neighborhood minima arrangements, convexity is an essential and fascinating property of the nonlinear SVM classifier. As per (Shave-Taylor and Cristian, 2004) "The nonattendance of neighborhood minima from the SVM algorithm marks the major dif-ference from conventional plans, for example, a neural network."(Rychetsky, 2001) "Exemplary learning frameworks like neural systems experience the ill effects of their hypothetical shortcoming eg: back-engendering often focalizes just too locally ideal arrangement. Here SVM's can give a generous change." According to the nonlinear SVM classifier SVM and ANN contrast fundamentally from practically identical methodologies, for example, neural systems: SVM preparing dependably finds a worldwide least, and their straightforward geometric translation gives fruitful soil to encourage examination." (O’sullivian, 2002) "In issues when direct choice hyper planes are never again possible, an effect space is spoken to into a component space bringing about a nonlinear classifier".

\subsection{Software Reliability:}

Programming Reliability is a fundamental component of programming quality and is one among the practically inescapable prospect of assessing the gauge of a product item. Programming industry perseveres through different difficulties in growing to a great degree solid programming. Measuring programming unwavering quality keeps on subsisting as a difficult issue since we don't have a full worry of the conduct of programming until the point when we practice it. There is no certain meaning of what perspectives identify with programming unwavering quality. We can't hold an appropriate way to gauge programming dependability, and a large portion of the perspectives identified with programming unwavering quality. Indeed, even the most evident item measurements have not unit-shape definition and we don't spare a specific strategy for each sort of programming. Foreseeing programming unwavering quality simply in view of the conduct and nature of the product.

\subsection{Machine Learning:}

ML is an approach which is centered on adapting naturally and enables PCs to develop and foresee the framework nature in view of past and the present disappointment information. Hence, it is very normal for programming specialists and analysts understand what specific technique is more significant to function admirably for a surrendered disappointment dataset and to what degree quantitatively. Here, we present an exact investigation of previously mentioned ML strategies for foreseeing programming unwavering quality in light of some modern datasets and explore about the precision and exhibitions of these models in reckoning the product dependability when connected to past disappointment week information. We likewise did a similar examination between total disappointment information and bury disappointment time information to research the sort of disappointment information more suitable for unwavering quality forecast.

\section{Software Reliability Prediction Using Ma- chine Learning Techniques:}

In this part, we investigate some notable and generally utilized machine learning strategies (ANNs, SVMs, CCNN, DTs and FIS) for the forecast of programming unwavering quality in view of the past disappointment conduct of programming associations. The capacity of ANNs to show complex non-direct connections and ability of approximating any quantifiable capacity make them alluring prospects for unraveling complex errands without bringing forth make an express model for the framework. Then again, SVM is a perusing framework, which builds an N-dimensional hyper plane that ideally isolates the information put into two classes. The fundamental pre-posture of SVM displaying is to characterize the ideal hyper plane that isolates groups of vector such that case with one classification of the reliant variable on one side of the plane and the cases with the other classification of the autonomous variable along the other position of the plane. The help vectors are the vectors close to the hyper plane. In this way SVMs can be connected as an option preparing technique for polynomial, spiral premise work and multilayer perception systems utilizing a portion part. In SVMs, the weights of the work are found by taking care of a quadratic programming issue with straight requirements, rather than by figuring out a non-arched, the unconstrained minimization issue as on account of preparing the neural net illustration. It is watched that SVM is proficient to sum up well, still in high dimensional spaces under little preparing test conditions. In this manner, SVMs have a superior ability of speculation because of the auxiliary hazard minimization standard.

\subsection{SVM (Support Vector Machines)}

"Support Vector Machine" (SVM) is a directed machine learning calculation which can be used for both arrangement and relapse problems. In any case, it is generally used as a piece of characterization issues. In this calculation, we plot every datum point as a point in $\mathrm{n}$-dimensional space (where $\mathrm{n}$ is the quantity of highlights you have) with the estimation of each element being the estimation of a particular facilitate. And after that, we perform order by finding the hyper-plane that separates the two classes exceptionally well.

SVM is a perusing framework, which builds $\mathrm{N}$-dimensional hyper planes that ideally separates the information put into two classes. Hyper plane in geometry is a subspace of one measurement not as much as its surrounding space. On the off chance that a space is 3more dimensional than its hyper plane is the 2-dimensional planes, while if the space is 2-dimensional, its hyper planes are the 1dimensional lines. A hyper plane is only an exposed inclination.

In the scientific model the whole human action of programming disappointments saw from separate al business and genuine tasks are used to the SVM show utilizing aggregate number of recognized programming disappointments as contributions for foreseeing future disappointment conduct of the product. One time the model has been prepared and learnt the innate inside property of the product come up short process, it can be utilized for the expectation of programming dependability sensibly (Ping and Hong 2006). The information focuses for preparing the SVM display (Pradeep Kumar, Yogesh Singh) [12]. 


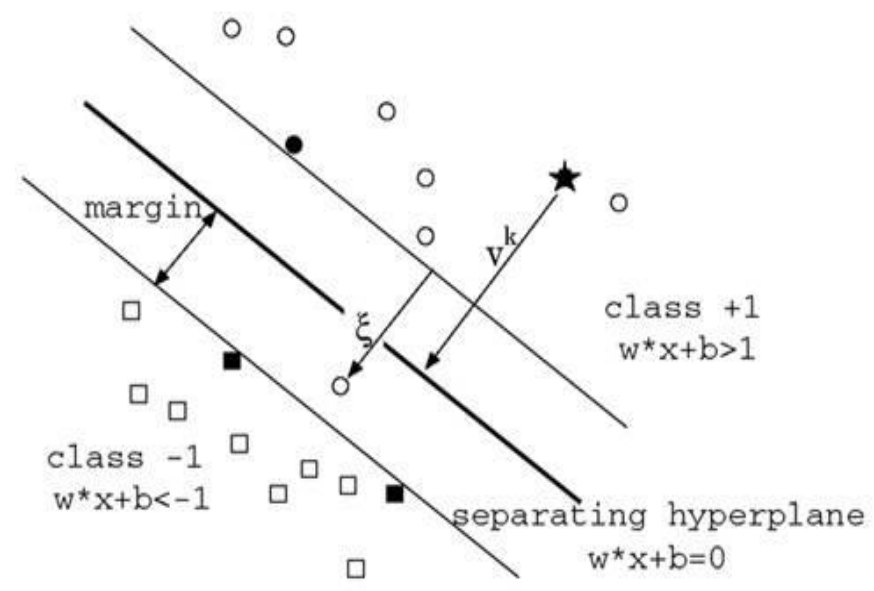

\subsection{Artificial Neural Network}

'Neural systems have seen a blast of exuberance in the form of the most recent couple of years and are as a rule, effectively connected over an uncommon range of issue spaces, in regions as assorted as a fund, solution, designing, topography and material science.' Statsoft.com [2010] it all began route in 1943 when McCullock and Pitts demonstrated that neuron can have two nations and that those countries could be reliant on some limit esteem. They displayed first simulated neuron demonstrate as indicated by Rojas [2005]. Numerous new and more refined models have been introduced since. McCullock and Pitt's revelation opened entryway for canny machines.

ANN works in a similar fashion to the human head, if the human mind were as mere as a CPU desktop with the utilization of the algorithm we wonder. In all likelihood, every human being would have ended up being identical someday or the other. For each, or the other situations, every one of us might have assumed the similar decisions. What could be the differentiating factor responsible for creating such diversity among humans is all about the human nerves might be one or the other react similar in places. I believe what distinguishes the human mind from a mere car. A simple machine set of algorithms, which switches the inputs into outputs. In such situations same inputs always had to the same outputs when a machine is involved other than the human brain comes up with multiple outputs purely because the involvement of neurons and are huge, reacts differently upon situations.

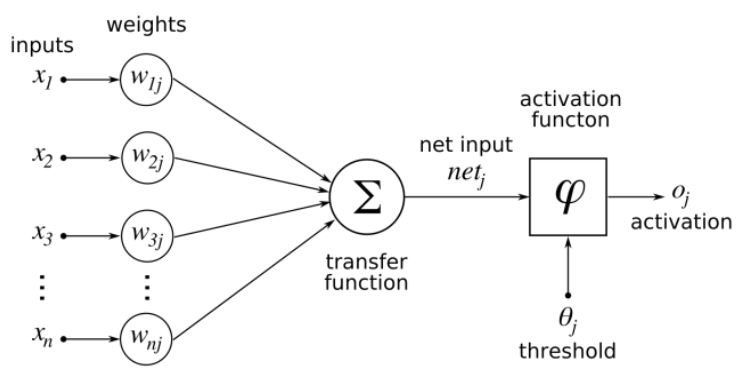

\subsection{Working of ANN}

Consider a topological network and which is connected through arrows headed toward right and they represent a connection between two neurons and indicate the pathway for the flow of information. Each connection has a weight, an integer number that takes the sign between the two neurons.

If the network generates a poor or undesired output or any defect then the system alters the weights in order to improve subsequent effects. If no errors then the sum of the weights in association with the nodes is being played out and creates an optimized solution.

\section{Back ground / Related work:}

Mechanical learning is a figuring plan, there are three strategies in the public arena to recognize the ranges of work by various machine learning methods are Supervised learning, Unsupervised learning, Reinforcement learning. Regulated (watch and direct the operation of an undertaking or action) learning is a kind of calculations comprise of goal/result variable (subordinate variable) which is to be shouted from a crumpled arrangement of indicators (autonomous factors). The preparation factors proceed until the point that the model accomplishes a coveted layer of precision of the preparation information. Illustration: relapse, choice tree, arbitrary woodland, KNN, strategic Regression, and so on. Applications: confront identification, signature acknowledgment, customer revelation, character acknowledgment, and growth diagnosis, medicate screening and so forth. Unsupervised learning (not done or acting under supervision). In these calculations we don't keep any goal or result variable to gauge or figure. It is utilized for bunching populace in various gatherings. Which is broadly utilized for portioning customers in various gatherings for particular intercession? Case: Apriority calculation, k-implies application information mining, content mining. Support taking in (the activity of the way toward fortifying): Using some remotely to advance utilizing the calculation the machine is set up to make particular determinations. It works: the machine is presented to a domain where it trains itself persistently utilizing the trail or blunder, this machine gains from part involvement and endeavours to get the most ideal information to make exact business decision. Example: Markov Decision Process.

\subsection{Research Background}

Our system for programming unwavering quality forecast utilizing machine learning procedures is presented specifically in our further procedures. The Waikato Environment for Knowledge Analysis (Weka) is a far reaching apparatus comprising of Java class libraries is accustomed to experiencing DTs. Alive is a free open-source suite made out of a few workplaces of the frail class library that were utilized to lead the investigations actualized on a Windows XP framework with a Web perusing capacity.

\section{The Metrics Used for Model Evaluation}

Correlation coefficient: Correlation coefficient measures the agreement of predictions with the real grade. This statistic suggests that how closely actual and predicted values are correlated.

Mean absolute error (MAE): It is the quantity depends on the prediction of minimum error that is to the actual subjects.MAE assesses the models output for each observation to check the recommended model gives the efficient output and tend to over or under estimate. The forecast and observed values are the model's outcomes from $\mathrm{n}$ observations.

Root mean squared error (RMSE): It is often used to compute the difference between values guessed by a prototype and the values really observed from the domain that has been modeled.

Precision: Precision is defined as the number of categories that are predicted correctly, divided by the total number of classes ROC curve ROC curve defined as a plot of sensitivity on the ycoordinate versus its (1-specificity) on the $\mathrm{x}$ coordinate.

ROC: It is an efficient process of measuring the performance of forecast models. Thus, Area under the ROC Curve (AUC) is a combined measure of sensitivity and specificity.

F-Measure: It is a way of combining recall and precision scores into a single measure of public presentation presented as follows: F-measure $=29$ recall 9 precision/ (recall? Precision). 
Sensitivity: Sensitivity also called recall rate that estimates the proportion of real places which are exactly identified. It measure the proportion of negatives, which are effectively identified as the ratio of No. of true negatives to the (no. Of true negatives? The ordinal number of false positives) $\mathrm{p}$ value $\mathrm{p}$ values are applied.

Linear regression: we can't always draw a straight job that passes through every data-level, but we can find such a task that comes closer to most of the data points. Hence the position represents the estimated average change in $\mathrm{Y}$ (failure rate) when $\mathrm{X}$ (testing period) increases by one unit used in our study.

\section{Empirical datasets}

\begin{tabular}{|c|l|l|}
\hline Dataset & Dataset Name & $\begin{array}{l}\text { Count of De- } \\
\text { tected Errors }\end{array}$ \\
\hline 1. & Real time command and control system & 135 \\
\hline 2. & Real time Command and control system & 54 \\
\hline 3. & Real time command and Control system & 38 \\
\hline 4. & Real time command and control system & 53 \\
\hline 5. & Real time Commercial system & 831 \\
\hline 6. & Commercial subsystem & 73 \\
\hline 14C & Real time System & 36 \\
\hline 17 & Military system & 38 \\
\hline 27 & Military system & 41 \\
\hline SS1A & Operating System & 112 \\
\hline SS1B & Operating System & 375 \\
\hline SS1C & Operating System & 277 \\
\hline SS2 & Time sharing System & 192 \\
\hline SS3 & Word Processing System & 278 \\
\hline SS4 & Operating System & 196 \\
\hline
\end{tabular}

\section{Expected Outcomes:}

\begin{tabular}{|l|l|l|l|l|l|}
\hline $\begin{array}{l}\text { Da- } \\
\text { taset }\end{array}$ & $\begin{array}{l}\text { Correla- } \\
\text { tion } \\
\text { Coeffi- } \\
\text { cients }\end{array}$ & $\begin{array}{l}\text { Mean } \\
\text { Abso- } \\
\text { lute } \\
\text { Error }\end{array}$ & $\begin{array}{l}\text { Trad- } \\
\text { ing } \\
\text { RMSB }\end{array}$ & $\begin{array}{l}\text { Testing } \\
\text { RMSB }\end{array}$ & $\begin{array}{l}\text { No. } \\
\text { of } \\
\text { Sup- } \\
\text { port } \\
\text { Vec- } \\
\text { tors }\end{array}$ \\
\hline 1. & 0.9989 & 1.9812 & 2.4963 & 2.6423 & 90 \\
\hline 2 & 0.9965 & 0.9681 & 1.292 & 2.5888 & 48 \\
\hline 3. & 0.9866 & 1.3230 & 1.7212 & 2.5692 & 26 \\
\hline 4. & 0.9882 & 1.9003 & 2.3417 & 2.5897 & 51 \\
\hline 5. & 0.9996 & 5.2684 & 7.3184 & 13.0850 & 741 \\
\hline 6. & 0.9982 & 1.9485 & 2.4525 & 3.6771 & 50 \\
\hline $14 C$ & 0.9850 & 1.3078 & 1.8158 & 2.3397 & 35 \\
\hline 17 & 0.9970 & 0.7425 & 0.8776 & 1.9292 & 15 \\
\hline 27 & 0.9932 & 0.9842 & 1.3172 & 4.5056 & 23 \\
\hline SS1A & 0.9988 & 1.1443 & 1.5331 & 1.9042 & 85 \\
\hline SS1B & 0.9986 & 3.0602 & 3.9785 & 5.4320 & 312 \\
\hline SS1C & 0.9986 & 3.0604 & 3.9734 & 5.1429 & 227 \\
\hline SS2 & 0.9987 & 2.2645 & 2.8062 & 3.1739 & 107 \\
\hline SS3 & 0.9921 & 2.4311 & 3.1103 & 3.1910 & 212 \\
\hline SS4 & 0.9989 & 2.0092 & 2.6201 & 3.1912 & 108 \\
\hline Tabl 8.1 & Summat & & & \\
\hline
\end{tabular}

Table 8.1 Summary of predictions using SVM

[Source: An empirical study of software reliability prediction using machine learning techniques]

\begin{tabular}{|l|l|l|l|l|}
\hline Dataset & $\begin{array}{l}\text { Correlation Coef- } \\
\text { ficient }\end{array}$ & $\begin{array}{l}\text { Slope of } \\
\text { LR }\end{array}$ & $\begin{array}{l}\text { P } \\
\text { value }\end{array}$ & RMSE \\
\hline 1. & 0.9212 & 0.6562 & 0.0000 & 13.1465 \\
\hline 2 & 0.8223 & 0.9681 & 0.0000 & 5.2802 \\
\hline 3. & 0.9086 & 0.7502 & 0.0006 & 2.5072 \\
\hline 4. & 0.8821 & 0.6615 & 0.0000 & 3.4367 \\
\hline 5. & 0.9671 & 0.7485 & 0.0000 & 9.7645 \\
\hline 6. & 0.9094 & 0.7646 & 0.0000 & 5.4921 \\
\hline $14 \mathrm{C}$ & 0.8845 & 0.8864 & 0.0001 & 2.8437 \\
\hline 17 & 0.8611 & 0.6867 & 0.0000 & 2.9742 \\
\hline 27 & 0.8928 & 0.6897 & 0.0000 & 2.9742 \\
\hline SS1A & 0.9885 & 0.8737 & 0.0000 & 3.3393 \\
\hline SS1B & 0.9710 & 0.8642 & 0.0000 & 7.5528 \\
\hline SS1C & 0.9807 & 0.8848 & 0.0000 & 9.5414 \\
\hline
\end{tabular}

\begin{tabular}{|l|l|l|l|l|}
\hline SS2 & 0.9765 & 0.8646 & 0.0000 & 8.3735 \\
\hline SS3 & 0.9857 & 0.9995 & 0.0000 & 7.2242 \\
\hline SS4 & 0.9320 & 0.0176 & 0.1105 & 9.6953 \\
\hline
\end{tabular}

Table 8.2: Summary of predictions for different data sets using back propagation neural network

[Source: An empirical study of software reliability prediction using machine learning techniques]

\section{Discussion}

1. The improvement of ANNs took after a heuristic way, with application and broad exploratory way, with applications and broad experimentation going before hypothesis. Interestingly, the improvement of SVM's included some speculation, then usage and tests.

2. A huge preferred standpoint of SVMs is that sooner or later ANN can experience the ill effects of neighborhood minima, the solution for an SVM is worldwide and one of a kind.

3. The two more preferences of SVMS are that they have a basic geometric translation and contributed a meager arrangement. Unlink ANN's the computational many-sided qualifications of SVMs are that have a straightforward geometric understanding and given a scanty arrangement.

4. "They contrast drastically from similar methodologies, for example, neural systems: SVM is preparing dependably locate a worldwide least, and their straightforward geometric translation gives fruitful ground to further examination."

5. Most frequently Gaussain parts are utilized, when they came about the SVM approach consequently fathoms the system multifaceted nature Problem, the size of the shrouded layer is brought as the after effect of the concealed neurons and bolster vectors compares to each other, and so the inside issue of RBF system is likewise gone down, as the bolster vector fills in as the premise work focuses.

6. "While the weight, rot term is an imperative viewpoint for getting great speculation with regards to neural system for relapse, the margin assumes a comparative part in characterization issue."

7. In comparison with customary multilayer discernment neural systems that accept the ominous effects of the presence of neighborhood minima arrangements, convexity is a lively and fascinating property of the nonlinear SVM classifier.

8. The nonappearance of neighborhood minima from the SVM calculation denotes the significant takeoff from conventional fabrics, for instance, neural systems."

\section{Acknowledgement}

The authors wish to thank people who helped with the study or preparation of the paper. The authors may want to thank anyone who had helpful discussions with them or contributed less tangible concepts.

\section{References}

Praman Deep Singh and Anuradha Chug, "Software Defect Prediction Analysis using Machine Learning Algorithms", 2017.

[1] IEEE Transactions on Software Engineering,Vol.43,No.1,January 2011. 
[2] Lina Chato*, Shahab Tayeb and Shahram Latifi," A Genetic Algorithm to Optimize the Adaptive Support Vector Regression Model for Forecasting the Reliability of Diesel Engine Systems " 2017.

[3] Akshi Kumar, Rajat Chugh, Rishab Girdhar and Simran Aggarwa," Classification of errors in Web Applications using Machine Learning" 2017.

[4] Harsh Lal and Gaurav Pahwa," Root Cause Analysis of Software Bugs using Machine Learning Techniques"2017.

[5] Alweshah, M., Ahmed, W., \& Aldabbas, H. (2016).'Evolution of Software Reliability Growth Models: A Comparison of AutoRegression and Genetic Programming Models",2015.

[6] Ruchika Malhotra," A Systematic Review of Machine Learning Techniques for Software Fault Prediction", 2016.

[7] Sankardas Roy1, Jordan DeLoach2, Yuping Li3, Nic Herndon2, Doina Caragea2, Xinming Ou 3, Venkatesh Prasad Ranganath2, Hongmin Li2, and Nicolais Guevara2 "Experimental Study with Real-world Data for Android App Security Analysis using Machine Learning",2015.

[8] Ramakanta Mohanty and M. R. Patra," Application of Machine learning techniques to Predict software reliability", 2010.

[9] Kuldeep Singh Kaswan, Sunita Choudhary and Kapil Sharma,"Software Reliability Modeling uses Soft Computing Techniques: Critical Review", 2015.

[10] Martin Shepperd, David Bowes and Tracy Hall," Researcher Bias: The Use of Machine Learning in Software Defect Prediction”, 2014

[11] T. Hall, S. Beecham, D. Bowes, D. Gray, and S. Counsell, "A systematic literature review on fault prediction performance in software engineering,", 2012

[12] Chih-Chung Chang and Chih-Jen Lin,'LIBSVM: A Library for Support Vector Machines", last Updated 2013.

[13] Pradeep Kumar and Yogesh Singh," An empirical survey of software reliability prediction using machine learning techniques", 2012.

[14] Malhotra R, Kaur A, Singh Y , "Empirical validation of object oriented metrics for predicting fault proneness at different severity levels using support vector machines", 2011 .

[15] Malhotra R, Singh Y, Kaur A "Comparative analysis of regression and machine learning methods for predicting fault proneness models", 2009.

[16] H. Pham, "System Software Reliability", Reliability Engineering Series, Springer, 2006

[17] Ji, S., Xu, W., Yang, M., Yu, K., “3D Convolutional Neural Networks for Human Action Recognition. IEEE Transactions on Pattern Analysis and Machine Intelligence” 2013. 